TAIWANESE JOURNAL OF MATHEMATICS

Vol. 13, No. 2A, pp. 459-466, April 2009

This paper is available online at http://www.tjm.nsysu.edu.tw/

\title{
WEAK AND WEAK* TOPOLOGIES AND BRODSKII-MILMAN'S THEOREM ON HYPERSPACES
}

\author{
Thakyin Hu and Jui-Chi Huang
}

\begin{abstract}
Let $K$ be a weakly compact, convex subset of a Banach space $X$ with normal structure. Brodskii and Milman proved that there exists a point $p \in K$ which is fixed under all isometries of $K$ onto $K$. Suppose now $W C C(X)$ is the collection of all non-empty weakly compact convex subsets of $X$. We shall define a certain weak topology $\mathcal{T}_{w}$ on $W C C(X)$ and have the above-mentioned result extended to the hyperspace $\left(W C C(X), \mathcal{T}_{w}\right)$
\end{abstract}

\section{INTRODUCTION}

Banach Contraction Principle and Schauder-Tychonof Theorem were published in the early 1900's. These theorems have important applications to various branches of mathematics. Suppose $K$ is a weakly compact, convex subset with normal structure of a Banach space, Brodskii and Millman [3] proved that there exists a point $p \in K$ which is fixed under all isometries of $K$ onto $K$, and Browder and Kirk ([4], [11]) proved that every non-expansive mapping of $K$ into $K$ has a fixed point. It is the main purpose of this paper to extend Brodskii-Milman's theorem to the hyperspace $W C C(X)$, where $X$ is a Banach space and $W C C(X)$ is the collection of all non-empty weakly compact convex subsets of $X$.

\section{Notations and PReliminaries}

Let $X$ be a Banach space, $X^{*}$ its topological dual and $B C C(X)$ be the collection of all non-empty bounded, closed convex subsets of $X$. For $A, B \in$ $B C C(X)$, define $N(A ; \varepsilon)=\{x \in X: d(x, a)=\|x-a\|<\varepsilon$ for some $a \in A\}$ and $h(A, B)=\inf \{\varepsilon>0: A \subset N(B ; \varepsilon)$ and $B \subset N(A ; \varepsilon)\}$, equivalently,

Received May 2, 2007, accepted September 1, 2007.

Communicated by Mau-Hsiang Shih.

2000 Mathematics Subject Classification: 54A05, 54A20, 54B20, 47H10.

Key words and phrases: Weak topology, Weak* topology, Brodskii-Milman's theorem, Hyperspace. 
$h(A, B)=\max \left\{\sup _{x \in A} d(x, B), \sup _{x \in B} d(x, A)\right\}$. Then $h$ is known as the Hausdorff metric and $(B C C(X), h)$ is known as the hyperspace over $X$. If $\operatorname{dim}(X)<\infty$ and $A_{n} \in B C C(X)$ is a bounded sequence (i.e. there exists $M<\infty$ such that $h\left(A_{n},\{0\}\right) \leq M$ for all $\left.n=1,2, \ldots\right)$, Blaschke [2] proved that $\left\{A_{n}\right\}$ has a subsequence $\left\{A_{n_{k}}\right\}$ such that $\left\{A_{n_{k}}\right\}$ converges to some $A \in B C C(X)$. DeBlasi and Myjak [2] introduced the concept of weak convergence of a sequence in $B C C(X)$ and they proved an infinite dimensional version of Blaschke's theorem. Other notions of weak convergence of bounded, closed, convex sets have been studied by other mathematicians $([1,13])$. Let $W C C(X)$ be the collection of all non-empty weakly compact convex subsets of $X$ and $C C(X)$ be the collection of all non-empty compact, convex subsets of $X$. For general $X$, we have $C C(X) \varsubsetneqq W C C(X) \varsubsetneqq B C C(X)$. If $X$ is reflexive, we have $W C C(X)=B C C(X)$. If $\operatorname{dim}(X)<\infty$, we have $C C(X)=W C C(X)=B C C(X)$. Weak topologies have been introduced on the hyperspaces $C C(X), W C C(X)$ and extensions of certain fixed point theorems are obtained ([7]-[11]). Suppose now $W^{*} C C\left(X^{*}\right)$ is the collection of all non-empty weak* compact, convex subsets of $X^{*}$. Because of the interplay between $X$ and $X^{*}$, the notion of weak topology on $W C C(X)$ leads us naturally to consider the concept of weak* topology on $W^{*} C C\left(X^{*}\right)$. And we shall prove in the sequel that Brodskii-Milman's theorem can be extended to the hyperspaces $W C C(X)$ and $W^{*} C C\left(X^{*}\right)$. To continue our discussion, we let $\mathbb{Z}$ denote the complex plane and $C C(\mathbb{Z})$ the collection of all non-empty compact, convex subsets of $\mathbb{Z}$. First, observe that for each $x^{*} \in X^{*}$, the weak continuity and linearity of $x^{*}$ imply that for each $A \in W C C(X)$ (i.e., $A$ is a weakly compact, convex subset of $X$ ), we have $x^{*}(A) \in C C(\mathbb{Z})$ (i.e., $x^{*}(A)$ is a compact, convex subset of the complex plane $\mathbb{Z}$ ). Thus each $x^{*}$ maps the space $W C C(X)$ into $C C(\mathbb{Z})$. Similarly each $x \in X$ maps the space $W^{*} C C\left(X^{*}\right)$ into $C C(\mathbb{Z})$.

\section{Lemma 1.}

(a) Suppose A, $B \in W C C(X)$. Then $h\left(x^{*}(A), x^{*}(B)\right) \leq\left\|x^{*}\right\| h(A, B)$ for each $x^{*} \in X^{*}$.

(b) Suppose $A^{*}, B^{*} \in W^{*} C C\left(X^{*}\right)$. Then $h\left(x\left(A^{*}\right), x\left(B^{*}\right)\right) \leq\|x\| h\left(A^{*}, B^{*}\right)$ for each $x \in X$.

Proof. Let $h(A, B)<r$. Then $A \subset N(B ; r)$ and $B \subset N(A ; r)$. Hence for each $a \in A$, there exists $b \in B$ such that $\|a-b\|<r$ and consequently, $\left\|x^{*}(a)-x^{*}(b)\right\| \leq\left\|x^{*}\right\|\|a-b\| \leq\left\|x^{*}\right\| \cdot r$, which in turn implies that $x^{*}(A) \subset$ $N\left(x^{*}(B) ;\left\|x^{*}\right\| r\right)$. Similarly, $x^{*}(B) \subset N\left(x^{*}(A) ;\left\|x^{*}\right\| r\right)$. Thus $h\left(x^{*}(A), x^{*}(B)\right) \leq$ $\left\|x^{*}\right\| h(A, B)$, and the proof is complete.

Suppose now $A, B \in W C C(X)$ with $B \not \subset A$, then there exists $b \in B$ but $b \notin A$. It follows from Hahn-Banach theorem that there exists $x^{*} \in X^{*}$ and real 
numbers $r_{1}, r_{2}$ such that $\operatorname{Re} x^{*}(a)<r_{1}<r_{2}<\operatorname{Re} x^{*}(b)$ for all $a \in A$. Thus $\left|x^{*}(b)-x^{*}(a)\right| \geq\left|\operatorname{Re} x^{*}(b)-\operatorname{Re} x^{*}(a)\right|>r_{2}-r_{1}$ for all $a \in A$ and consequently $x^{*}(b) \notin x^{*}(A)$ which implies $h\left(x^{*}(B), x^{*}(A)\right)>0$ (i.e. $x^{*}(B) \neq x^{*}(A)$ ). The above brief discussion yields the following Lemma 2.

\section{Lemma 2.}

(a) $A=B$ if and only if $x^{*}(A)=x^{*}(B)$ for each $x^{*} \in X^{*}$, where $A, B \in$ $W C C(X)$.

(b) $A^{*}=B^{*}$ if and only if $x\left(A^{*}\right)=x\left(B^{*}\right)$ for each $x \in X$, where $A^{*}, B^{*} \in$ $W^{*} C C\left(X^{*}\right)$.

Definitions. Recall that the weak topology $\tau_{w}$ on $X$ is defined to be the weakest topology which makes each $x^{*}:\left(X, \tau_{w}\right) \rightarrow(\mathbb{Z},|\cdot|)$ continuous. It follows from Lemma 1 that each $x^{*}:(W C C(X), h) \rightarrow(C C(\mathbb{Z}), h)$ is continuous. Thus we may define $\mathcal{T}_{w}$ to be the weakest topology on the hyperspace $W C C(X)$ such that each $x^{*}:\left(W C C(X), \mathcal{T}_{w}\right) \rightarrow(C C(\mathbb{Z}), h)$ is continuous. Similarly, $\mathcal{T}_{w}^{*}$ is defined to be the weakest topology which makes each $x:\left(W^{*} C C\left(X^{*}\right), \mathcal{T}_{w}^{*}\right) \rightarrow(C C(\mathbb{Z}), h)$ continuous. A typical weak neighborhood $\left(\mathcal{T}_{w}\right.$-neighborhood) of $A \in W C C(X)$ is denoted by $\mathcal{W}\left(\overline{\left.A ; x_{1}^{*}, \ldots, x_{n}^{*} ; \varepsilon\right)}=\left\{B \in W C C(X): h\left(x_{i}^{*}(B), x_{i}^{*}(A)\right)<\varepsilon\right.\right.$ for $i=1,2, \ldots, n\}$, and a weak ${ }^{*}$ neighborhood $\left(\mathcal{T}_{w}^{*}\right.$-neighborhood) of $A^{*} \in$ $W^{*} C C\left(X^{*}\right)$ is denoted by $\overline{\mathcal{W}^{*}\left(A^{*} ; x_{1}, \ldots, x_{n} ; \varepsilon\right)}=\overline{\left\{B^{*} \in W^{*} C C\left(X^{*}\right):\right.} h\left(x_{i}\left(B^{*}\right)\right.$, $\left.x_{i}\left(A^{*}\right)\right)<\varepsilon$ for $\left.i=1,2, \ldots, n\right\}$. Also for $A, B \in W C C(X)$ and $\alpha \in \mathbb{Z}$, it follows from the continuity of addition and scalar multiplication that $A+B$ and $\alpha A$ belong to $W C C(X)$. Thus a subset $\mathcal{K} \subset W C C(X)$ is defined to be convex if for each $A_{1}, A_{2}, \ldots, A_{n} \in \mathcal{K}$ and $\alpha_{1}, \alpha_{2}, \ldots, \alpha_{n} \in[0,1]$ with $\sum_{i=1}^{n} \alpha_{i}=1$, we have $\sum_{i=1}^{n} \alpha_{i} A_{i} \in \mathcal{K} . \mathcal{K}$ is said to have normal structure ([6], [14]) if for each convex $\mathcal{M} \subset \mathcal{K}$, and $\mathcal{M}$ is not a singleten, then $\mathcal{M}$ has a non-diametral point (i.e., there exists $A \in \mathcal{M}$ such that $\sup \{h(A, B): B \in \mathcal{M}\}<\operatorname{diam} \mathcal{M}=\sup \{h(A, B)$ : $A, B \in \mathcal{M}\}$.

Let $\bar{X}=\{\bar{x}=\{x\}: x \in X\}$ (i.e. $\bar{X}$ is the hyperspace consisting of singletons). Then $(\bar{X}, h)$ may be identified with $(X,\|\cdot\|)$, and $\left(\bar{X}, \mathcal{T}_{w}\right)$ may be identified with $\left(X, \tau_{w}\right)$ naturally. Thus theorems on hyperspaces are extensions of their counterparts on original underlying spaces. We remind our readers that we use small letters to denote elements of the underlying Banach spaces $X$ and $X^{*}$; capital letters to denote subsets of $X$ and $X^{*}$ as well as elements of the hyperspaces $W C C(X)$ and $W^{*} C C\left(X^{*}\right)$; script letters to denote subsets of hyperspaces. Thus $B[0, r]=\{x \in X \mid\|x\| \leq r\}$ and $B^{*}[0, r]$ are closed balls of $X$ and $X^{*}$; $\mathcal{B}[0, r]=\{A \in W C C(X): h(A,\{0\}) \leq r\}$ and $\mathcal{B}^{*}[0, r]$ are closed balls of $W C C(X)$ and $W^{*} C C\left(X^{*}\right)$, respectively. 
We shall need the following Lemma 3 which has been noted in ([6], [7]) and is easily verifiable.

Lemma 3. Let $A, B, C, D \in W C C(X)$ and $\alpha \in \mathbb{Z}$. Then

(a) $h(\alpha A, \alpha B)=|\alpha| h(A, B)$, and

(b) $h(A+B, C+D) \leq h(A, C)+h(B, D)$.

\section{Main Results}

We shall use the Uniform Boundedness Principle and Hahn-Banach Theorem to establish some fundamental properties of the hyperspaces. We prove that weakly compact subsets of $W C C(X)$ are weakly closed and bounded and weak* compact subsets of $W^{*} C C\left(X^{*}\right)$ are weak* closed and bounded. Also, we will prove that closed balls $\mathcal{U}[A, \delta]$ and $\mathcal{U}\left[A^{*}, \delta\right]$ are weakly closed and weak*-closed respectively. These properties are essential tools to establish the main theorem of this paper, namely, extension of Brodskii-Milman's theorem to the hyperspaces.

\section{Theorem 1.}

(a) A weakly compact subset $\mathcal{K} \subset W C C(X)$ is weakly closed and bounded.

(b) A weak ${ }^{*}$-compact subset $\mathcal{K}^{*} \subset W^{*} C C\left(X^{*}\right)$ is weak ${ }^{*}$-closed and bounded.

Proof. We shall prove only part (b) since the proof of part (a) is essentially the same. Suppose $\mathcal{K}^{*}$ is weak*-compact. Then $\mathcal{K}^{*}$ is weak ${ }^{*}$-closed since the weak*topology $\mathcal{T}_{w}^{*}$ is Hausdorff. Also for each $x \in X$, it follows from the definition that $x:\left(W^{*} C C\left(X^{*}\right), \mathcal{T}_{w}^{*}\right) \rightarrow(C C(\mathbb{Z}), h)$ is continuous and hence $x\left(\mathcal{K}^{*}\right)=\left\{x\left(A^{*}\right):\right.$ $\left.A^{*} \in \mathcal{K}^{*}\right\}$ is a compact subset of the metric space $(C C(\mathbb{Z}), h)$, which implies the existence of some $M_{x}<\infty$ such that $\sup \left\{h\left(x\left(A^{*}\right), x(\{0\})\right): A^{*} \in \mathcal{K}^{*}\right\} \leq M_{x}<$ $\infty$. Note that $h\left(x\left(A^{*}\right), x(\{0\})\right)=\sup \left\{\left\|x\left(a^{*}\right)\right\|: a^{*} \in A^{*}\right\}$. Thus if we set $K^{*}=$ $\bigcup_{A^{*} \in \mathcal{K}^{*}} A^{*}=\bigcup_{A^{*} \in \mathcal{K}^{*}}\left\{a^{*}: a^{*} \in A^{*}\right\} \subseteq X^{*}$, we have $\sup \left\{h\left(x\left(A^{*}\right), x(\{0\})\right): A^{*} \in\right.$ $\left.\mathcal{K}^{*}\right\}=\sup _{A^{*} \in \mathcal{K}^{*}}\left[\sup \left\{\left\|x\left(a^{*}\right)\right\|: a^{*} \in A^{*}\right\}\right]=\sup \left\{\left\|x\left(a^{*}\right)\right\|: a^{*} \in K^{*}\right\} \leq M_{x}<\infty$. Consequently, $K^{*} \subset X^{*}$ is a collection of linear functionals that is pointwise bounded at each $x \in X$. It follows now from the uniform boundedness principle that $K^{*}$ is a bounded subset of $X^{*}$, i.e., $\sup \left\{\left\|a^{*}\right\|: a^{*} \in K^{*}\right\} \leq N<\infty$ for some $N$. now for each $A^{*} \in \mathcal{K}^{*}$, we have $h\left(A^{*},\{0\}\right)=\sup \left\{\left\|a^{*}\right\|: a^{*} \in A^{*}\right\} \leq N$, since $A^{*} \subset K^{*}$. Thus $\mathcal{K}^{*}$ is a bounded subset of $\left(W^{*} C C\left(X^{*}\right), h\right)$ and the proof is complete.

\section{Theorem 2.}

(a) The closed ball $\mathcal{U}[A, \delta]$ of the hyperspace $(W C C(X), h)$ is weakly closed (i.e. $\mathcal{T}_{w}$-closed), 
(b) the closed ball $\mathcal{U}\left[A^{*}, \delta\right]$ of the hyperspace $\left(W^{*} C C\left(X^{*}\right), h\right)$ is weak ${ }^{*}$-closed (i.e., $\mathcal{T}_{w}^{*}$-closed $)$.

Proof. We shall prove part (b) only since the proof of part (a) is similar. Let $B^{*} \notin \mathcal{U}\left[A^{*}, \delta\right]$ with $h\left(A^{*}, B^{*}\right)=\delta+r$ where $r>0$. Since $h\left(A^{*}, B^{*}\right)=$ $\max \left\{\sup _{x^{*} \in A^{*}} d\left(x^{*}, B^{*}\right), \sup _{x^{*} \in B^{*}} d\left(x^{*}, A^{*}\right)\right\}$, we shall consider the cases $h\left(A^{*}, B^{*}\right)=$ $\sup _{x^{*} \in A^{*}} d\left(x^{*}, B^{*}\right)$ and $h\left(A^{*}, B^{*}\right)=\sup _{x^{*} \in B^{*}} d\left(x^{*}, A^{*}\right)$ separately.

Case 1. Suppose $h\left(A^{*}, B^{*}\right)=\sup _{x^{*} \in A^{*}} d\left(x^{*}, B^{*}\right)$, then there exists $a_{0}^{*} \in A^{*}$ such that $d\left(a_{0}^{*}, B^{*}\right) \geq h\left(A^{*}, B^{*}\right)-\frac{r}{3}$. It follows that for each $b^{*} \in B^{*},\left\|a_{0}^{*}-b^{*}\right\| \geq$ $d\left(a_{0}^{*}, B^{*}\right) \geq h\left(A^{*}, B^{*}\right)-\frac{r}{3}=(\delta+r)-\frac{r}{3}=\delta+\frac{2 r}{3}>\delta+\frac{r}{3}$ and hence $b^{*} \notin N\left[a_{0}^{*} ; \delta+\right.$ $\left.\frac{r}{3}\right]$ showing that $N\left[a_{0}^{*} ; \delta+\frac{r}{3}\right] \cap B^{*}=\varnothing$, where both $N\left[a_{0}^{*} ; \delta+\frac{r}{3}\right]$ and $B^{*}$ are weak*compact convex sets. It follows now from the Hahn-Banach theorem that there exists $x \in X$ and real numbers $r_{1}, r_{2}$ such that $\operatorname{Re} x\left(b^{*}\right)<r_{1}<r_{2}<\operatorname{Re} x\left(x^{*}\right)$ for $b^{*} \in B^{*}$ and $x^{*} \in N\left[a_{0}^{*} ; \delta+\frac{r}{3}\right]$. Let $\varepsilon=\frac{r_{2}-r_{1}}{2}$. Suppose now $A_{k}^{*} \in \mathcal{U}\left[A^{*}, \delta\right]$, we have $h\left(A_{k}^{*}, A^{*}\right) \leq \delta$ which in turn implies the existence of some $a_{k}^{*} \in A_{k}^{*}$ with $\left\|a_{k}^{*}-a_{0}^{*}\right\|<\delta+\frac{r}{3}$ or $a_{k}^{*} \in N\left(a_{0}^{*} ; \delta+\frac{r}{3}\right) \subset N\left[a_{0}^{*} ; \delta+\frac{r}{3}\right]$. Thus $\left|x\left(a_{k}^{*}\right)-x\left(b^{*}\right)\right| \geq$ $\left|\operatorname{Re} x\left(a_{k}^{*}\right)-\operatorname{Re} x\left(b^{*}\right)\right|>r_{2}-r_{1}>\varepsilon$ for all $b^{*} \in B^{*}$, i.e. $x\left(a_{k}^{*}\right) \notin N\left(x\left(B^{*}\right) ; \varepsilon\right)$ which in turn implies $x\left(A_{k}^{*}\right) \not \subset N\left(x\left(B^{*}\right) ; \varepsilon\right)$ and hence $h\left(x\left(A_{k}^{*}\right), x\left(B^{*}\right)\right) \geq \varepsilon$. Thus $A_{k}^{*} \notin \mathcal{W}\left(B^{*} ; x ; \varepsilon\right)$ proving that each $B^{*} \notin \mathcal{U}\left[A^{*}, \delta\right]$ has a weak*-neighborhood $\mathcal{W}\left(B^{*} ; x ; \varepsilon\right)$ disjoint from $\mathcal{U}\left[A^{*}, \delta\right]$. Thus the complement of $\mathcal{U}\left[A^{*}, \delta\right]$ is weak*open and hence $\mathcal{U}\left[A^{*}, \delta\right]$ is weak*-closed.

Case 2. Suppose $h\left(A^{*}, B^{*}\right)=\sup _{x^{*} \in B^{*}} d\left(x^{*}, A^{*}\right)$. It follows that there exists $b_{0}^{*} \in B^{*}$ such that $d\left(b_{0}^{*}, A^{*}\right) \geq h\left(A^{*}, B^{*}\right)-\frac{r}{3}$. Let $D^{*}=\bigcup_{a^{*} \in A^{*}} N\left[a^{*} ; \delta+\frac{r}{3}\right]=$ $A^{*}+N\left[0 ; \delta+\frac{r}{3}\right]$ where both $A^{*}$ and $N\left[0 ; \delta+\frac{r}{3}\right]$ are weak*-compact, convex and hence $D^{*}$ is also weak*-compact, convex. Now for each $x^{*} \in D^{*}$, there exists $a^{*} \in A^{*}$ with $\left\|x^{*}-a^{*}\right\| \leq \delta+\frac{r}{3}$. Thus $\left\|a^{*}-b_{0}^{*}\right\| \leq\left\|a^{*}-x^{*}\right\|+\left\|x^{*}-b_{0}^{*}\right\|$ which in turn implies that $\left\|x^{*}-b_{0}^{*}\right\| \geq\left\|a^{*}-b_{0}^{*}\right\|-\left\|a^{*}-x^{*}\right\| \geq d\left(b_{0}^{*}, A^{*}\right)-\left\|a^{*}-x^{*}\right\| \geq$ $h\left(A^{*}, B^{*}\right)-\frac{r}{3}-\left\|a^{*}-x^{*}\right\| \geq(\delta+r)-\frac{r}{3}-\left(\delta+\frac{r}{3}\right)=\frac{r}{3}$. Consequently, $d\left(b_{0}^{*}, D^{*}\right) \geq \frac{r}{3}$ and we may apply Hahn-Banach theorem to get some $x \in X$ and real numbers $r_{1}, r_{2}$ such that $\operatorname{Re} x\left(x^{*}\right)<r_{1}<r_{2}<\operatorname{Re} x\left(b_{0}^{*}\right)$ for all $x^{*} \in D^{*}$, which implies $\left|x\left(b_{0}^{*}\right)-x\left(x^{*}\right)\right| \geq\left|\operatorname{Re} x\left(b_{0}^{*}\right)-\operatorname{Re} x\left(x^{*}\right)\right|>r_{2}-r_{1}>\frac{r_{2}-r_{1}}{2}=\varepsilon$ for all $x^{*} \in D^{*}$. Next, if $A_{k}^{*} \in \mathcal{U}\left[A^{*}, \delta\right]$ implies $h\left(A_{k}^{*}, A^{*}\right) \leq \delta<\delta+\frac{r}{3}$ and hence $A_{k}^{*} \subset N\left(A^{*} ; \delta+\frac{r}{3}\right) \subset D^{*}$. Consequently, $\left|x\left(b_{0}^{*}\right)-x\left(a_{k}^{*}\right)\right| \geq r_{2}-r_{1}>\varepsilon$ for each $a_{k}^{*} \in A_{k}^{*}$, which implies $x\left(B^{*}\right) \not \subset N\left(x\left(A_{k}^{*}\right), \varepsilon\right)$. Thus $h\left(x\left(A_{k}^{*}\right), x\left(B^{*}\right)\right) \geq \varepsilon$ showing that $A_{k}^{*} \notin \mathcal{W}\left(B^{*} ; x ; \varepsilon\right)$. Therefore, the complement of $\mathcal{U}\left(A^{*}, \delta\right]$ is weak*open and hence $\mathcal{U}\left[A^{*}, \delta\right]$ is weak ${ }^{*}$-closed and the proof is complete. 


\section{Theorem 3.}

(a) Suppose $\mathcal{K}$ is a non-empty, weakly compact (i.e. $\mathcal{T}_{w}$-compact), convex subset of $W C C(X)$ and $\mathcal{K}$ has normal structure. Then $\mathcal{K}$ contains a point $A_{0}$ which is fixed under all isometries of $\mathcal{K}$ onto $\mathcal{K}$.

(b) Suppose $\mathcal{K}^{*}$ is a non-empty, weak ${ }^{*}$-compact (i.e. $\mathcal{T}_{w}^{*}$-compact), convex subset of $W^{*} C C\left(X^{*}\right)$ and $\mathcal{K}^{*}$ has normal structure. Then $\mathcal{K}^{*}$ contains a point $A_{0}^{*}$ which is fixed under all isometries of $\mathcal{K}^{*}$ onto $\mathcal{K}^{*}$.

Proof. We shall prove part (a) only. Let $\mathcal{F}=\{T: \mathcal{K} \rightarrow \mathcal{K} \mid T$ is a surjective isometry\}. Observe that $T \in \mathcal{F}$ implies $T^{-1} \in \mathcal{F}$ since $T: \mathcal{K} \rightarrow \mathcal{K}$ is $1-1$, onto. We may now use Zorn's Lemma to obtain a set $\mathcal{K}_{0} \subset \mathcal{K}$ which is minimal with respect to being non-empty, weakly compact, convex and invariant under $T$ (i.e., $\left.T\left(\mathcal{K}_{0}\right) \subset \mathcal{K}_{0}\right)$ for each $T \in \mathcal{F}$. If $\mathcal{K}_{0}$ consists of a single element, we are done. Otherwise $0<\operatorname{diam}\left(\mathcal{K}_{0}\right)=d$. Since $\mathcal{K}_{0}$ is weakly compact, it follows from Theorem 1(a) that $\operatorname{diam}\left(\mathcal{K}_{0}\right)=d<\infty$. Since $\mathcal{K}$ has normal structure, it follows that $\mathcal{K}_{0}$ has a non-diametral point, i.e., there exists $A_{0} \in \mathcal{K}_{0}$ such that $\sup \left\{h\left(A_{0}, A\right): A \in \mathcal{K}_{0}\right\}=d_{1}<d$. Let $\mathcal{K}_{1}=\mathcal{K}_{0} \cap\left(\bigcap_{A \in \mathcal{K}_{0}} \mathcal{U}\left[A, d_{1}\right]\right)$. Since $A_{0} \in \mathcal{K}_{1}$, therefore $\mathcal{K}_{1} \neq \varnothing . \mathcal{K}_{1}$ is convex since all sets involved are convex. Also each $\mathcal{U}\left[A, d_{1}\right]$ is weakly closed by Theorem 2 . Thus $\mathcal{K}_{1}$ is weakly closed and hence weakly compact since it is contained in the weakly compact set $\mathcal{K}_{0}$. Since $T\left(\mathcal{K}_{0}\right) \subset \mathcal{K}_{0}$ for each $T \in \mathcal{F}$, for any given $B \in \mathcal{K}_{0}$, we have $T^{-1}(B) \in \mathcal{K}_{0}$ and $T\left(T^{-1}(B)\right)=B$ showing that $T\left(\mathcal{K}_{0}\right)=\mathcal{K}_{0}$ for each $T \in \mathcal{F}$. Next, we claim that $T\left(\mathcal{K}_{1}\right) \subset \mathcal{K}_{1}$ for each $\mathcal{K}_{1}$. To prove our claim, we let $B \in \mathcal{K}_{1}$ and $T \in \mathcal{F}$ be given, then for any $A \in \mathcal{K}_{0}$, we have $T^{-1}(A) \in \mathcal{K}_{0}$ and $h(T(B), A)=$ $h\left(T(B), T\left(T^{-1}(A)\right)=h\left(B, T^{-1}(A)\right) \leq d_{1}\right.$. Consequently, $h(T(B), A) \leq d_{1}$ for any $A \in \mathcal{K}_{0}$ and hence $T(B) \in \mathcal{K}_{0} \cap\left\{\bigcap_{A \in \mathcal{K}_{0}} \mathcal{U}\left[A, d_{1}\right]\right\}=\mathcal{K}_{1}$ and the claim is proved. Thus $\mathcal{K}_{1}$ is a non-empty weakly compact, convex subset of $\mathcal{K}_{0}$ which is invariant under each $T \in \mathcal{F}$. Moreover, $d_{1}<d$ implies that $\mathcal{K}_{1} \varsubsetneqq \mathcal{K}_{0}$. That is a contradiction to the minimality of $\mathcal{K}_{0}$ and the theorem is proved.

Suppose $X$ is uniformly convex or $\operatorname{dim}(X)<\infty$. Then it is well-known that $X$ has normal structure ([6], [14]). We shall prove that the hyperspace $C C(X)$ has normal structure if $\operatorname{dim}(X)<\infty$.

Theorem 4. Suppose $\operatorname{dim}(X)<\infty$. Then $C C(X)$ has normal structure.

Proof. It follows from Blaschke's theorem that every closed and bounded subset $\mathcal{K} \subset(C C(X), h)$ is compact. Also every set $\mathcal{K}$ and its closure has the same diameter. Thus it is sufficient to prove that if $\mathcal{K} \subset C C(X)$ is $h$-compact and convex with $\operatorname{diam}(\mathcal{K})=d>0$, then $\mathcal{K}$ has a non-diametral point $A_{0} \in \mathcal{K}$ (i.e. $\sup \left\{h\left(A_{0}, A\right): A \in \mathcal{K}\right\}=d_{1}<d$ ). Assume the contrary, then every point 
of $\mathcal{K}$ is a diametral point. Let $A_{1} \in \mathcal{K}, A_{1}$ is diametral and $\mathcal{K}$ is compact implies the existence of some $A_{2} \in \mathcal{K}$ such that $h\left(A_{1}, A_{2}\right)=d$. By convexity of $\mathcal{K}$, $\left(A_{1}+A_{2}\right) / 2 \in \mathcal{K}$ and $\left(A_{1}+A_{2}\right) / 2$ is diametral. Let $A_{3} \in \mathcal{K}$ be such that $h\left(\left(A_{1}+\right.\right.$ $\left.\left.A_{2}\right) / 2, A_{3}\right)=d$. Since $d=h\left(\left(A_{1}+A_{2}\right) / 2, A_{3}\right)=h\left(\left(A_{1}+A_{2}\right) / 2,\left(A_{3}+A_{3}\right) / 2\right) \leq$ $\frac{1}{2} h\left(A_{1}, A_{3}\right)+\frac{1}{2} h\left(A_{2}, A_{3}\right) \leq \frac{1}{2} d+\frac{1}{2} d=d$. It follows that $h\left(A_{1}, A_{3}\right)=h\left(A_{2}, A_{3}\right)=$ $d$. Inductively, if $A_{1}, A_{2}, \ldots, A_{n} \in \mathcal{K}$ has been chosen such that $h\left(A_{i}, A_{j}\right)=d$ where $i, j \in\{1,2, \ldots, n\}$ and $i \neq j$. Then $\left(A_{1}+A_{2}+\cdots+A_{n}\right) / n \in \mathcal{K}$ is diametral implies the existence of $A_{n+1} \in \mathcal{K}$ such that $h\left(A_{i}, A_{n+1}\right)=d$ for $i=1,2, \ldots, n$. Consequently $\left\{A_{i}\right\}$ is an infinite sequence of $\mathcal{K}$ such that $h\left(A_{i}, A_{j}\right)=d$ for $i \neq j$. Thus $\left\{A_{i}\right\}$ is an infinite sequence that has no convergent subsequence. That is a contradiction to the compactness of $\mathcal{K}$, and hence the theorem is proved.

\section{REFERENCES}

1. G. Bear, Topologies on Closed and Closed Convex Sets, Mathematics and its Applications, 268, Kluwer Academic Publishers, Dordrecht, 1993.

2. W. Blaschke, 'Kreis und Kugel', Chelsea Publishing Company, New York, 1949.

3. M. S. Brodskii and D. P. Milman, On the center of a convex set, Dokl. Akad. Nauk. SSSR N. X., 59 (1948), 837-840.

4. F. E. Browder, Nonexpansive nonlinear operators in a Banach space, Proc. Nat. Acad. Sci. U.S.A., 54 (1965), 1041-1044.

5. F. S. DeBlasi and J. Myjak, Weak convergence of convex sets, Arch. Math., 47 (1986), 448-456.

6. K. Goebel and W. A. Kirk, Topics in Metric Fixed Point Theory, Cambridge University Press, 1990.

7. T. Hu and J.-C. Fang, Weak topology and Browder-Kirk's theorem on hyperspace, J. Math. Anal. Appl. (2007), doi:10.1016/j.jmaa.2006.12.078.

8. T. Hu and J.-C. Huang, Weak topology and Markov-Kakutani theorem on hyperspace, Publ. Math. Debrecen, 53 (1998), 113-117.

9. T. Hu and W. S. Heng, An extension of Markov-Kakutani's fixed point theorem, Indian J. Pure Appl. Math., 32 (2001), 899-902.

10. T. Hu, J.-C. Huang and B. E. Rhoades, A general principle for Ishikawa iterations for multi-valued mappings, Indian J. Pure Appl. Math., 28 (1997), 1091-1098.

11. T. Hu and J.-C. Huang, Weak and strong convergence in the hyperspace $C C(X)$, Taiwanese J. Math., accepted.

12. W. A. Kirk, A fixed point theorem for mappings which do not increase distances, Amer. Math. Monthly, 72 (1965), 1004-1006.

13. Y. Sonntag and C. Zalinescu, Scalar convergence of convex sets, J. Math. Anal. Appl., 164 (1992), 219-241. 
14. E. Zeidler, Nonlinear Functional Analysis and its Applications I: Fixed Point Theorems, Springer, New York, 1986.

Thakyin $\mathrm{Hu}$

Department of Mathematics,

Tamkang University,

Tamsui, Taipei,

Taiwan 25137, R.O.C.

Jui-Chi Huang

Center for General Education,

Technology and Science Institute of Northern Taiwan,

No. 2, Xue Yuan Road, Peito,

Taipei 112, Taiwan, R.O.C.

E-mail: juichi@tsint.edu.tw

juichi.h0207@msa.hinet.net 